\title{
Article
}

\section{Targeting the IL-6/STAT3 Signalling Cascade to Reverse Tamoxifen Resistance in Estrogen Receptor Positive Breast Cancer}

\author{
Ho Tsoi, Ellen P. S. Man, Ka Man Chau and Ui-Soon Khoo *(D)
}

Citation: Tsoi, H.; Man, E.P.S.; Chau, K.M.; Khoo, U.-S. Targeting the IL-6/STAT3 Signalling Cascade to Reverse Tamoxifen Resistance in Estrogen Receptor Positive Breast Cancer. Cancers 2021, 13, 1511. https://doi.org/10.3390/cancers 13071511

Academic Editor: Patrizia Limonta

Received: 26 February 2021

Accepted: 22 March 2021

Published: 25 March 2021

Publisher's Note: MDPI stays neutral with regard to jurisdictional claims in published maps and institutional affiliations.

Copyright: (c) 2021 by the authors. Licensee MDPI, Basel, Switzerland. This article is an open access article distributed under the terms and conditions of the Creative Commons Attribution (CC BY) license (https:// creativecommons.org/licenses/by/ $4.0 /)$.

\author{
Department of Pathology, Li Ka Shing Faculty of Medicine, The University of Hong Kong, Hong Kong, China; \\ tsoiho@hku.hk (H.T.); ellenman@hku.hk (E.P.S.M.); 15435717@life.hkbu.edu.hk (K.M.C.) \\ * Correspondence: uskhoo@pathology.hku.hk
}

Simple Summary: This study identifies the molecular mechanisms through which BQ323636.1 can enhance IL-6 and IL-6R expression, which leads to the activation of STAT3 and the development of tamoxifen resistance in ER+ breast cancer. We demonstrated a statistically significant association of IL-6R with tamoxifen resistance; patients with high IL-6R expression had poorer survival outcome. In vitro and in vivo studies confirmed that targeting IL-6R with Tocilizumab reduced tamoxifen resistance, providing the basis for potential use for disease management

\begin{abstract}
Breast cancer is the most common female cancer. About $70 \%$ of breast cancer patients are estrogen receptor $\alpha(\mathrm{ER} \alpha)$ positive (ER + ) with tamoxifen being the most commonly used anti-endocrine therapy. However, up to $50 \%$ of patients who receive tamoxifen suffer recurrence. We previously identified BQ323636.1 (BQ), a novel splice variant of NCOR2, can robustly predict tamoxifen resistance in ER+ primary breast cancer. Here we show that BQ can enhance IL-6/STAT3 signalling. We demonstrated that through interfering with NCOR2 suppressive activity, BQ favours the binding of ER to IL-6 promoter and the binding of NF-kB to IL-6 receptor (IL-6R) promoter, leading to the up-regulation of both IL-6 and IL-6R and thus the activation of STAT3. Knockdown of IL-6R could compromise tamoxifen resistance mediated by BQ. Furthermore, Tocilizumab (TCZ), an antibody that binds to IL-6R, could effectively reverse tamoxifen resistance both in vitro and in vivo. Analysis of clinical breast cancer samples confirmed that IL-6R expression was significantly associated with BQ expression and tamoxifen resistance in primary breast cancer, with high IL-6R expression correlating with poorer survival. Multivariate Cox-regression analysis confirmed that high IL-6R expression remained significantly associated with poor overall as well as disease-specific survival in ER+ breast cancer.
\end{abstract}

Keywords: breast cancer; interleukin-6; interleukin-6 receptor; tamoxifen resistance; Tocilizumab; STAT3

\section{Introduction}

Breast cancer is the most common cancer in women worldwide, with nearly 1.7 million new cases diagnosed in 2012. There are five distinct molecular subtypes of breast cancer: luminal A and B, human epidermal growth factor receptor 2 (HER2)-enriched, basal-like, and claudin-low, with the last two being subcategories of triple-negative breast cancer [1]. Each subtype can be targeted differently with systemic therapy. The available systemic therapies include endocrine therapy, targeted biologic treatment, and chemotherapy. Luminal A and B breast cancers are ER-positive subtypes of breast cancer in which estrogen regulates and mediates cell growth. About $70 \%$ of breast cancer patients are ER positive $(E R+)$. The estrogen receptor- $\alpha(E R \alpha)$ is a major driver of tumor growth in $\mathrm{ER}+$ breast cancer. ER $\alpha$ cooperates with several other transcription factors to control gene expression and ultimately, tumour growth [2]. ER $\alpha$-pathway-targeted treatments (e.g., 
aromatase inhibitors, tamoxifen, and fulvestrant) are the standard of care for patients with this disease [3,4]. However, up to $50 \%$ of patients who receive tamoxifen suffer recurrence. Recurrence may be due to the development of de novo or acquired resistance to tamoxifen [5]. Lack of ER $\alpha$ expression is the dominant mechanism of de novo resistance to tamoxifen [6]. Epigenetic changes in the ER $\alpha$ gene may contribute to the development of tamoxifen resistance [5]. Alteration in signalling cascades are essential for development of acquired resistance to tamoxifen. One such mechanism involves cross-talk between ER $\alpha$ and growth factor-mediated signalling pathways [7]. Furthermore, alternative Splicing (AS), which is a predominant mechanism for generating distinct $m$ RNA isoforms from a single gene, also plays an important role in cancer development and treatment [8].

Interleukin-6 (IL-6) is a pleiotropic cytokine that plays a central role in both normal human physiology and disease [9]. IL-6 binds to the receptor complex consisting of IL-6 binding type I transmembrane glycoprotein termed IL-6 receptor- $\alpha$ (IL-6R, CD126) and the type I transmembrane signal transducer protein gp130 (CD130) to activate downstream Janus kinases (JAKs), which subsequently activate Signal transducer and activator of transcription 3 (STAT3) through the phosphorylation of tyrosine 705 [10]. IL-6/STAT3 signalling has been shown to play an important role in tumor progression in many solid tumor types by inducing epithelial-to-mesenchymal transition and angiogenesis [11,12]. IL-6 has been implicated as a driver in pre-clinical models of ER+ breast cancer, and high IL-6 serum and tumor levels have been associated with aggressiveness and poor outcome in patients [13-15]. Therefore, the IL-6/STAT3 pathway is a pharmacological target for treating human diseases, cancer included. Multiple approaches have been employed to target this pathway pre-clinically as well as clinically, including targeting of upstream JAKs (e.g., Ruxolitinib), direct targeting of STAT3 phosphorylation and activation (e.g., OPB compounds), and downregulating STAT3 expression (e.g., AZD9150) [16]. Tocilizumab (TCZ; Actemra ${ }^{\circledR}$ ), which is a humanized monoclonal antibody that binds and neutralizes IL-6R, resulting in the inhibition of IL-6-mediated signalling, has been used clinically to treat rheumatoid arthritis [17].

Expression of IL-6 is governed by Nuclear factor- $\kappa$ B (NF- $\kappa$ B) [18], which is a family of inducible transcription factors, which regulates a large array of genes involved in different processes of the immune and inflammatory responses [19]. This family is composed of five structurally related members, including NF-kB1 (p50), NF-kB2 (p52), RelA (p65), RelB, and c-Rel, which mediates transcription of target genes by binding to a specific DNA element, $\kappa B$ enhancer, as various hetero- or homo-dimers [20]. NF- $\kappa$ B is commonly specifically referred to a p50-p65 heterodimer, which is one of the most avidly forming dimers and is the major NF-KB complex in most cells [20]. NCOR2 (SMRT) is a key corepressor protein that functions to repress transcription of various transcription factors [21]. Studies have demonstrated that NCOR2 exhibited an interaction with p50 and suppressed the DNA transcriptional activity of p65 [22,23].

We previously identified a novel splice variant of NCOR2 named BQ323636.1 (BQ) and found it to be associated with tamoxifen resistance [24]. Our group examined the expression of BQ by immunohistochemistry in 2095 cases of primary breast cancer in tissue microarray (TMA) from Hong Kong and the United Kingdom. Of the 1271 cases assessed, those with nuclear BQ overexpression showed statistically significant association with poorer survival outcome and tamoxifen resistance, demonstrating that nuclear BQ expression in primary breast cancer could be a robust biomarker for predicting tamoxifen resistance in ER+ breast cancer [25]. Mechanistically, BQ competed with NCOR2 to functionally interfere with the formation of NCOR2 gene co-repressor complex; thus, BQ overexpression compromised the function of NCOR2 in repressing gene expression [25].

In the current study, we demonstrate that BQ overexpression could enhance the expression of IL-6 and IL-6R, which in turn activates the IL-6/STAT3 pathway to mediate tamoxifen resistance in breast cancer. Through in vivo study, we confirmed the clinical significance of IL-6R in breast cancer. By targeting IL-6R, tamoxifen resistance could be 
reversed both in vitro and in vivo. Our study thus elucidated a potential method for managing tamoxifen resistance in breast cancer.

\section{Materials and Methods}

\subsection{Cell Culture and Stable Cell Lines Establishment}

Human breast cancer cell lines MCF-7 and ZR-75, both which are ER+ and tamoxifen sensitive, were purchased from ATTC and reauthenticated by short tandem repeat profiling [24]. LCC2, a tamoxifen-resistant cell line derived from MCF-7, and AK-47, a tamoxifen resistant cell line derived from ZR-75 cells, were kindly provided by Dr. Robert Clarke (Georgetown University Medical School, Washington, DC, USA) [26]. MCF-7 and LCC2 cells were cultured and maintained in Dulbecco's Modified Eagle Medium (DMEM, Gibco, Gaithersburg, MD, USA) supplemented with 10\% fetal bovine serum (Gibco, Gaithersburg, MD, USA) and 1\% penicillin/streptomycin (Gibco, Gaithersburg, MD, USA). ZR-75 and AK-47 cells were grown in Improved Minimum Essential Medium (IMEM, Gibco, Gaithersburg, MD, USA) with the addition of $10 \% \mathrm{FBS}$ and $1 \% \mathrm{P} / \mathrm{S}$. MCF-10A was purchased from ATCC and cultured in Mammary Epithelial Cell Growth Medium (Lonza, Morristown, NJ, USA) supplemented by Bovine Pituitary Extract (Lonza), Human Epidermal Growth Factor (Lonza, Morristown, NJ, USA), Hydrocortisone, and $100 \mathrm{ng} / \mathrm{mL}$ cholera toxin.

MCF-7 and ZR-75 were transfected with pcDNA3.1 or pcDNA3.1-BQ to establish control cell lines and $\mathrm{BQ}$ overexpressing cell lines respectively. The stable cell lines were selected and maintained in the culture medium with $0.5 \mathrm{mg} / \mathrm{mL}$ of G418 (Gibco, Gaithersburg, MD, USA). IL-6R Human shRNA Plasmid Kit (TL312161; Origene, Rockville, MD, USA) was employed. MCF-7, ZR-75, LCC2, and AK-47 were transfected with plasmid, which expressed non-targeting shRNA (shCtrl), shRNA1 targeting IL-6R (shIL-6R.1; TL312161A), and shRNA2 targeting IL-6R (shIL-6R.2; TL312161B). The stable cell lines were selected and maintained in the culture medium with $1 \mu \mathrm{g} / \mathrm{mL}$ of puromycin (Gibco, Gaithersburg, MD, USA). IL-6 CRISPR/Cas9 KO Plasmid (sc-400390; Santa Cruz Biotechnology, Dallas, TX, USA) and IL-6 HDR Plasmid (sc-400390-HDR; Santa Cruz Biotechnology) were employed to generate IL-6 knockout (KO) cell lines. MCF-7-BQ and ZR-75-BQ were cotransfected with IL-6 CRISPR/Cas9 KO Plasmid and IL-6 HDR Plasmid. The stable cell lines were selected and maintained in the culture medium with $1 \mu \mathrm{g} / \mathrm{mL}$ of puromycin (Gibco, Gaithersburg, MD, USA) and $0.5 \mathrm{mg} / \mathrm{mL}$ of G418 (Gibco, Gaithersburg, MD, USA). All the cell lines were cultured in a tissue culture incubator with $5 \% \mathrm{CO}_{2}$ at $37^{\circ} \mathrm{C}$. All cell lines were confirmed free of mycoplasma (service provided by core facilities, The University of Hong Kong).

\subsection{Chemicals}

Human IL-6 Recombinant Protein (RP-8619; Invitrogen, Waltham, MA, USA) and bovine serum albumin (BSA; J10857-36; Thermo Scientific, Waltham, MA, USA) were dissolved in double-distilled water. STAT3 Inhibitor VI S3I-201 (sc-204304; Santa Cruz Biotechnology, Dallas, TX, USA) was dissolved in DMSO. Tocilizumab (TCZ; Actemra ${ }^{\circledR}$; Roche, Switzerland) was used and diluted in saline. $\beta$-Estradiol ( $\mathrm{E}_{2} ; \mathrm{E} 8875 ;$ Sigma, St. Louis, MO, USA) was dissolved in 100\% ethanol. NF-kB inhibitor p-XSC (ab142600; Abcam, Cambridge, UK) was dissolved in DMSO. Tamoxifen (TAM/4-OHT; H6278; Sigma, St. Louis, MO, USA) was dissolved into $100 \%$ ethanol (EtOH).

\subsection{Cell Viability Assay}

MTT assay (3-(4,5-Dimethylthiazol-2-yl)-2,5-Diphenyltetrazolium Bromide; M6494; Invitrogen) was performed according to the manufacturer's instructions. Absorbance was recorded by Tecan Infinite F200 plate-reader. Clonogenic assay was performed by staining the cells with $0.01 \%$ of crystal violet (C0775; Sigma, St. Louis, MO, USA). Any colony with more than 50 cells was regarded as one colony. 


\subsection{ELISA Assay}

IL-6 Human ELISA Kit (EH2IL6; Invitrogen, Waltham, MA, USA) was used according to the instruction manual. Absorbance at $450 \mathrm{~nm}$ was recorded by Tecan infinite F200 platereader.

\subsection{Gene Silencing, Plasmids, $q P C R$, Gene Expression Analysis and Promoter Analysis}

siBQ.1 (5'-CUU CUC CAG GUU CUC UGC AUG-3') and siBQ.2 (5'-CUC CAG GUU CUC UGC AUG CGC-3') were purchased (Sigma). Negative control siRNA (siCtrl; 4390843; ThermoFisher, Waltham, MA, USA) and IL-6 siRNA (siIL-6; s7311; ThermoFisher, Waltham, MA, USA) were employed. Ten pmol of the siRNA was used. Oligofectamine (12252011; Invitrogen, Waltham, MA, USA) was used for the delivery of the siRNA. TRIzol reagent (15596026; Invitrogen, Waltham, MA, USA) was used for total RNA extraction following manufacturer's protocol. Up to $0.5 \mu \mathrm{g}$ total RNA were reverse transcribed into cDNA by SuperScript III reverse transcriptase (18080093; Invitrogen, St. Louis, MO, USA) following the manufacturer's protocol. Overexpression of NCOR2 was mediated by transfection of pCMV6_NCOR2 (RC212113; Origene, MD, USA) using Lipofectamine 2000 (11668-019; Invitrogen, Waltham, MA, USA). Applied Biosystems 7900HT was used. qPCR was employed to determine the relative expression of genes. $\mathrm{RT}^{2}$ Profiler ${ }^{\mathrm{TM}} \mathrm{PCR}$ Array Human Cancer PathwayFinder ${ }^{\mathrm{TM}}$ (PAHS-033Z; Qiagen, Germany) was used. Power SYBR Green Master Mix (A25742; Applied Biosystems, Foster City, CA, USA) was used. $\Delta \Delta C T$ method was used to determine relative gene expression level. Actin was used as the internal control. Untreated control was used as the reference. Pathway enrichment analysis was preformed using KEGG database. The following primers $\left(5^{\prime} \rightarrow 3^{\prime}\right)$ were used: actin-F (ATC GTG CGT GAC ATT AAG GAG AAG) and actin-R (AGG AAG GAA GGC TGG AAG AGT G); IL-6-F (ATA ACC ACC CCT GAC CCA AC) and IL-6-R (GAA CTC CTT AAA GCT GCG CA). IL-6R-F (AGA CAG GTG CGA AAG GAT GA) and IL-6R-R (TCT ACA GAC AAG CCC AGC AA) BQ-F (AAG GTG GAG CGC ATC GAG AAC) and BQ-R (GCA TCT GCT TCT CCA GGT TCT CTG); NCOR2-F (ACG AGG TGT CAG AGA TCA TCG A) and NCOR2-R (TGA TGA ACT TGA TGC GCT GCT). DNA sequence between +100 to -1000 was retrieved using The Eukaryotic Promoter Database on 10 June 2020 (https:/ / epd.epfl.ch//index.php) [27]. ER binding site and NF-kB1 binding site were examined in IL-6 and IL-6R promoters respectively. The location of transcription initiator was determined within the promoter region.

\subsection{Chromatin Immunoprecipitation (ChIP)}

Cell lysates were fixed for $10 \mathrm{~min}$ with $0.5 \%$ paraformaldehyde and suspended in $200 \mu \mathrm{L}$ of lysis buffer (20 mM Tris-Cl, pH 7.4, $150 \mathrm{mM} \mathrm{NaCl}, 5 \mathrm{mM} \mathrm{MgCl}_{2}$, and $0.5 \%$ Nonidet P-40). The suspended cells were lysed by sonication to yield DNA fragments of 0.1-5 kb using Diagenode BioruptorPico UltraSonication System (Centre for PanorOmic Sciences, HKU). Protein A agarose beads (20333; Thermo Scientific, Waltham, MA, USA) were precleared for $1 \mathrm{~h}$ at $4{ }^{\circ} \mathrm{C}$ in the presence of $100 \mu \mathrm{g} / \mathrm{mL}$ salmon sperm DNA (AM9680; Invitrogen, Waltham, MA, USA). Antigens were immunoprecipitated overnight at $4{ }^{\circ} \mathrm{C}$ with the following antibodies: anti-p50 (1:200; 13586; Cell signaling Technology, Danvers, MA, USA), Anti-ER $\alpha$ (1:200; 8644; Cell signaling Technology, Danvers, MA, USA). Ten percent of cell lysate was saved as input control. The agarose beads were washed in lysis buffer three times each for $10 \mathrm{~min}$ before elution. Cross-links were reversed by heating for $6 \mathrm{~h}$ at $65^{\circ} \mathrm{C}$. The DNA fragments were then eluted and purified by QIAquick PCR purification kit (28104; Qiagen, Germany). The purified DNA fragments were amplified by PCR, using the following primers $\left(5^{\prime} \rightarrow 3^{\prime}\right)$ : IL-6-ERE1-F (CAT GCC AAA GTG CTG AGT CA) and IL-6-ERE1-R (AGT GCA GCT TAG GTC GTC AT); IL-6-ERE2-F (CCC TCA CCC TCC AAC AAA GA) and IL-6-ERE2-R (GAG CTT CTC TTT CGT TCC CG); IL6R-P50-RE-F (AGT CCA AAC CGT TTC CTT GC); and IL6R-P50-RE-R (GGC GAT GTT CCT CTT ACC CT). 


\subsection{Tissue Microarray}

One-hundred-and-forty-one cases of breast cancer diagnosed between the years 1993-2003 with clinical follow up data were retrieved from the records of the Department of Pathology, Queen Mary Hospital of Hong Kong, with approval (UW 08-147) by the Institutional Review Board of The University of Hong Kong. The status of ER, PR, and HER2 were obtained from pathological reports from Queen Mary Hospital. ER, PR, and HER2 expression in the primary tumors were examined by IHC. Those cases with equivocal HER2 expression were further examined by FISH. Histological sections of these cases were reviewed by the pathologist; the representative paraffin tumor blocks were chosen as donor block for each case. Selected areas were marked for construction of tissue microarray (TMA) blocks. A total of 137 cases could be assessed (Table 1). Of these, there were $73 \mathrm{ER}+$ cases (Table 2) that had been given adjuvant tamoxifen treatment with available follow-up clinical data. Tamoxifen resistance is defined as patients who had been treated with tamoxifen in the adjuvant setting but subsequently developed disease relapse or distant metastases.

\subsection{Immunohistochemistry}

The IHC was performed as previously described [25]. Anti-BQ antibody (D-12; Versitech LTD, Telegraph Bay, Hong Kong) and anti-IL-6R antibody (A101411; Antibodies.com, Cambridge, UK) were diluted at 1:50 and 1:30 respectively. Aperio ImageScope ${ }^{\circledR}$ system (Leica Biosystems Aperio, Nußloch, Germany) was used to assess BQ323636.1 and IL-6R expression and scored by two independent individuals. For BQ staining, the intensities and percentages of nuclear staining were assessed using H-scoring system. $\mathrm{H}$-score $=(1 \times \%$ of cells stained at intensity category 1$)+(2 \times \%$ of cells stained at intensity category 2$)$ $+(3 \times \%$ of cells stained at intensity category 3$)$. As IL- $6 \mathrm{R}$ is localized to the cytoplasm, the intensities and percentages of cytoplasmic staining were assessed as follows: Intensity was scored as $0=$ none, $1=$ weak, $2=$ moderate, and $3=$ strong. The percentage of cells stained was scored as $1=$ less than $25 \%, 2=>25 \%$ to $50 \%, 3=>50 \%$ to $75 \%$, and $4=>75 \%$. The cytoplasmic score was calculated as the product of the percentage score and the intensity score. The median value of $\mathrm{H}$-score and cytoplasmic score were used to dichotomize the cut-off between low and high expression. These were 110 and 6 for BQ and IL-6R respectively.

\subsection{Luciferase Reporter Assay}

STAT3 Reporter Kit (79730; BPS Bioscience, San Diego, CA, USA) and NF-kB Reporter Kit (60614; BPS Bioscience, San Diego, CA, USA) were employed and Lipofectamine 2000 (11668-019; Invitrogen, Waltham, MA, USA) was used for the transfection. DualLuciferase $^{\circledR}$ Reporter Assay System was employed (E1910; Promega, Madison, WI, USA). Chemoluminance signal was recorded by Tecan infinite F200 plate reader.

\subsection{Preparation of Conditional Medium}

MCF-7, MCF-7-BQ, MCF-7-BQ siCtrl, MCF-7-BQ silL6, ZR-75, ZR-75-BQ, ZR-75-BQ siCtrl, ZR-75-BQ silL6, LCC2, and AK-47 cell lines were cultured in the medium (phenolred free) supplement with $10 \%$ of charcoal-stripped FBS (Gibco, Gaithersburg, MD, USA) and $1 \%$ penicillin/streptomycin (Gibco, Gaithersburg, ML, USA). After $72 \mathrm{~h}$, the medium was collected and centrifuged for $10 \mathrm{~min}$ at $4{ }^{\circ} \mathrm{C}$ with $4000 \mathrm{rpm}$ by Heraeus multifuge $\times 3$ FR centrifuge (rotor: 75003180; Thermo Scientific, Waltham, MA, USA) to remove cell debris. The conditional medium $(\mathrm{CM})$ was stored at $-20{ }^{\circ} \mathrm{C}$ in aliquots.

\subsection{Tamoxifen Response Assay}

The cells were treated with $5 \mu \mathrm{M}$ of tamoxifen (4-OHT; H6278; Sigma, St. Louis, MO, USA). 4-OHT was dissolved into $100 \%$ ethanol (EtOH). MTT and clonogenic assays were employed to determine the proportion of viable cells. 
Table 1. Clinical characterization of all breast cancer patients in the current study.

\begin{tabular}{|c|c|c|c|}
\hline Clinical Characteristic & Type & Cases & Percentage $(\%)$ \\
\hline Number of breast cancer patients & & 132 & \\
\hline Median Age & & 56 & \\
\hline \multicolumn{4}{|l|}{ T stage } \\
\hline & I & 16 & 12.1 \\
\hline & II & 25 & 18.9 \\
\hline & III & 5 & 3.8 \\
\hline & Missing & 86 & 65.2 \\
\hline \multicolumn{4}{|l|}{ Lymph Node status } \\
\hline & Positive & 62 & 47.0 \\
\hline & Negative & 56 & 42.4 \\
\hline & Missing & 14 & 10.6 \\
\hline \multicolumn{4}{|l|}{ Tumor Grade } \\
\hline & 1 & 19 & 14.4 \\
\hline & 2 & 29 & 22.0 \\
\hline & 3 & 71 & 53.8 \\
\hline & Missing & 13 & 9.8 \\
\hline \multicolumn{4}{|l|}{ Tumor Size } \\
\hline & $<2 \mathrm{~cm}$ & 37 & 28.0 \\
\hline & $\geq 2 \mathrm{~cm}$ & 53 & 40.2 \\
\hline & Missing & 42 & 31.8 \\
\hline \multicolumn{4}{|c|}{ Estrogen Receptor status } \\
\hline & Positive & 71 & 53.8 \\
\hline & Negative & 23 & 17.4 \\
\hline & Missing & 38 & 28.8 \\
\hline \multicolumn{4}{|c|}{ Progesterone receptor status } \\
\hline & Positive & 48 & 36.3 \\
\hline & Negative & 34 & 25.8 \\
\hline & Missing & 50 & 37.9 \\
\hline \multicolumn{4}{|l|}{ HER2 receptor status } \\
\hline & Positive & 33 & 25.0 \\
\hline & Negative & 34 & 27.3 \\
\hline & Missing & 50 & 47.7 \\
\hline \multicolumn{4}{|c|}{ Triple Negative status } \\
\hline & Positive & 12 & 9.10 \\
\hline & Negative & 68 & 51.5 \\
\hline & Missing & 52 & 39.4 \\
\hline
\end{tabular}


Table 2. Clinical characterization of ER+ breast cancer patients in the current study.

\begin{tabular}{|c|c|c|c|}
\hline Clinical Characteristic & Type & Cases & Percentage $(\%)$ \\
\hline Number of ER+ breast cancer patients & & 71 & \\
\hline Median Age & & 51 & \\
\hline \multicolumn{4}{|l|}{ T stage } \\
\hline & I & 8 & 11.3 \\
\hline & II & 18 & 25.3 \\
\hline & III & 3 & 4.2 \\
\hline & Missing & 42 & 59.2 \\
\hline \multicolumn{4}{|l|}{ Lymph Node status } \\
\hline & Positive & 37 & 52.1 \\
\hline & Negative & 28 & 39.4 \\
\hline & Missing & 6 & 8.5 \\
\hline \multicolumn{4}{|l|}{ Tumor Grade } \\
\hline & 1 & 13 & 18.3 \\
\hline & 2 & 21 & 29.6 \\
\hline & 3 & 36 & 50.7 \\
\hline & Missing & 1 & 1.4 \\
\hline \multicolumn{4}{|l|}{ Tumor Size } \\
\hline & $<2 \mathrm{~cm}$ & 22 & 31.0 \\
\hline & $\geq 2 \mathrm{~cm}$ & 32 & 45.1 \\
\hline & Missing & 17 & 23.9 \\
\hline \multicolumn{4}{|c|}{ Progesterone receptor status } \\
\hline & Positive & 47 & 66.2 \\
\hline & Negative & 12 & 16.9 \\
\hline & Missing & 12 & 16.9 \\
\hline \multicolumn{4}{|l|}{ HER2 receptor status } \\
\hline & Positive & 27 & 38.0 \\
\hline & Negative & 23 & 32.4 \\
\hline & Missing & 21 & 29.6 \\
\hline
\end{tabular}

\subsection{Western Blot and Co-Immunoprecipitation}

Cell pellets were lysed using cell lysis buffer prepared by mixing $1 \times$ Cell Lysis Buffer (9803; Cell Signaling Technology, Danvers, MA, USA), 10\% glycerol, cOmplete Mini, EDTA-free protease inhibitor cocktail (11873580001; Roche, Switzerland) and PhosSTOP EASYPack tablets (4906845001; Roche, Switzerland). Cell pellets were lysed in $200 \mu \mathrm{L}$ of IP buffer (20 mM Tris-Cl, pH7.4, 150 mM NaCl, 5 mM MgCl2, 0.5\% NP-40, 10\% glycerol). Twenty microliters was stored as input. The remaining cell lysates were divided into two portions, $90 \mu \mathrm{L}$ each. Anti-ER $\alpha$ (1:200; 8644; Cell signalling Technology, Danvers, MA, USA) and anti-NCOR2 (1:200; ab24551l; Abcam, Cambridge, UK) were added and incubated at $4{ }^{\circ} \mathrm{C}$ overnight with rotation. Anti-mouse/rabbit IgG was used as negative control. The immunoprecipitant was incubated with $50 \mu \mathrm{L}$ of Protein A/G agarose beads (20421; Thermo Scientific, Waltham, MA, USA) at room temperature for $2 \mathrm{~h}$ with rotation. The beads were washed three time with $1 \mathrm{~mL}$ of IP buffer, $10 \mathrm{~min}$ each. Protein A/G HRP (1:8000; 32490; Pierce, Rockford, IL, USA) was used as secondary antibody. The protein concentration of the cell lysates was determined by DC protein assay (1620177; BioRad, 
Hercules, CA, USA). SDS-polyacrylamide gels were made following the protocol for Western blot analysis. Twenty micrograms of total proteins were loaded in each of the wells of the gel. The proteins were transferred onto PVDF membrane (1620177; BioRad, Hercules, CA, USA). The signal was captured Amersham Imager 680 (GE Healthcare). Uncropped blots were shown in Figure S7. The follow antibodies were used: anti-HIS tag (1:4000; \#2366; Cell Signaling Technology); anti-BQ (1:500; D-12; Versitech Ltd., Telegraph Bay, Hong Kong); anti-STAT3 (1:1000; 9139; Cell Signaling Technology, Danvers, MA, USA); antipSTAT3 (1:1000; 9131, Cell Signaling Technology, Danvers, MA, USA); anti-IL-6R (1:2000; ab128008; Abcam, UK); anti-NCOR2 (1:1000; ab24551l; Abcam, UK); anti-p50 (1:2000; 13586; Cell Signaling Technology, Danvers, MA, USA), anti-p65 (1:1000; 8242; Cell Signaling Technology, Danvers, MA, USA); anti-tubulin (1:10,000; 2146; Cell Signaling Technology, Danvers, MA, USA); and anti-actin (1:10,000; sc-47778; Santa Cruz Biotechnology, Dallas, TX, USA). The following reagents were used to generate signal: anti-mouse HRP (1:5000; P0447; Dako, Denmark), anti-rabbit HRP (1:5000; P0260; Dako, Denmark).

\subsection{Xenograft}

Female nude mice, aged 5 to 6 weeks, were used for this study. $1 \times 10^{7}$ cells were mixed with Matrigel (356234; BD Bioscience, Franklin Lakes, NJ, USA) at a ratio of 1:1 and the $100 \mu \mathrm{L}$ cell mixture injected into the abdominal mammary fat pad of mice. When the tumors were palpable, mice were randomized into treatment and control groups where the treatment group received $0.5 \mathrm{mg}$ of 4-OHT tamoxifen (H6278; Sigma, St. Louis, MO, USA) dissolved in ethanol and diluted in peanut oil (P2144; Sigma, St. Louis, MO, USA), and TCZ $(1 \mathrm{mg} / \mathrm{Kg}$ and $2 \mathrm{mg} / \mathrm{Kg}$ ) diluted in saline, given by subcutaneous injection. The mice were treated twice a week for 8 weeks.

\subsection{Statistical Analysis}

All numerical data were processed in Excel (Microsoft), Prism5 (GraphPad) or SPSS25 (IBM). All data were expressed as mean \pm SD from at least three independent experiments. Mann-Whitney U test or Students' $t$ test were performed to compare the variables of the two sample groups. All tests were two-sided unless otherwise specified. The data were dichotomized into two groups including high or low expression using median expression level as cut-off. The correlations were analyzed by Chi-square tests. The expression levels of BQ, IL-6R were compared between different groups using Mann-Whitney U Rank test. Survival analyses were done by Kaplan-Meier estimates followed by Log-rank test and Cox regression model. $p$ value of less than 0.05 was considered statistically significant.

\section{Results}

\subsection{Overexpression of $B Q$ Enhanced IL-6/STAT3 Signalling Pathway}

Our previous studies had confirmed that BQ overexpression resulted in tamoxifen resistance in breast cancer cells both in vitro and in vivo [25]. In addition, we confirmed that tamoxifen-resistant cells have a higher ratio of BQ to NCOR2 (Figure S1A). To elucidate the molecular mechanisms by which $\mathrm{BQ}$ could contribute to the resistance, we employed Cancer PathFinder PCR array to determine the effect of BQ on the expression of 84 cancerrelated genes. The comparison was made between two pairs of cell lines, MCF-7-BQ versus MCF-7 and ZR-75-BQ versus ZR-75. From the expression profiles (Figure 1A and Table S1), we observe that $\mathrm{BQ}$ overexpression enhanced the expression of a certain subset of genes common for both MCF-7 and ZR-75. KEGG pathway enrichment analysis identified seven common pathways significantly enriched $(p<0.05)$ for both MCF-7 and ZR-75 (Figure 1B and Table S2), with the IL-6/STAT3 signalling pathway one of the significantly enriched pathways involved in both cell lines. qPCR independently validated that overexpression of BQ could enhance mRNA IL-6 expression in both of the cell lines (Figure 1C), whilst ELISA assay confirmed BQ overexpression could enhance the production of IL-6 (Figure 1D). IL-6 is known to enhance the activity of STAT3 through triggering phosphorylation on STAT3 [11]. We confirmed that BQ overexpression could indeed enhance the level of phos- 
phorylated STAT3 through Western blot (Figure 1E) and could enhance the transcriptional activity of STAT3 through luciferase reporter assay (Figure 1F). On the other hand, we designed two independent siRNA to reduce the expression of BQ in LCC2 cells (Figure 1G), which had no effect on NCOR2 mRNA expression (Figure S1B). Through STAT3 luciferase reporter assay, we found that down-regulation of BQ could suppress STAT3 activity in LCC2 (Figure 1H). Moreover, addition of IL-6 in LCC2 treated with siBQ.1 and siBQ.2 could rescue the activity of STAT3 (Figure S1C). Based on these findings, we confirmed that overexpression of BQ could modulate IL-6/STAT3 signalling pathway.

\subsection{Activation of IL-6/STAT3 Pathway Could Induce Tamoxifen Resistance}

We next confirmed that tamoxifen resistance could be induced by the activation of IL-6/STAT3 signalling. First, we collected conditional medium (CM) from MCF-7-BQ and ZR-75-BQ cells and used the CM to treat MCF-7 and ZR-75 together with tamoxifen. After $96-\mathrm{h}$, cell viability was determined by MTT assay. The results showed that the $\mathrm{CM}$ from BQ-overexpressing cells could induce the development of tamoxifen resistance (Figure 2A). Similarly, we collected CM from LCC2 and AK-47, which expressed high endogenous $\mathrm{BQ}$. As expected, the CM could induce tamoxifen resistance in MCF-7 and ZR-75 (Figure 2B). Next, we employed 20 pmol of siRNA against IL-6 or non-targeting siRNAs to treat MCF-7-BQ, ZR-75-BQ, LCC2, and AK-47 (Figure S2A). The culture medium from these siRNA-treated cells were collected and used to cultivate with MCF-7 and ZR-75 cells. ELISA assay confirmed that the IL-6 siRNA could reduce the production of IL-6 significantly (Figure 2C). The results from tamoxifen response assay showed that downregulation of IL- 6 could compromise the effect on the induction of tamoxifen resistance (Figure 2D). Moreover, knockout of IL-6 (Figure S2B) in MCF-7-BQ and ZR-75-BQ could reverse tamoxifen resistance as revealed by MTT (Figure 2E). Next, we employed recombinant IL-6 to test whether the treatment of IL-6 directly could induce tamoxifen resistance. We determined the optimal dosage of IL- 6 by examining its effect on cell viability and STAT3 activity. The results showed that $10 \mathrm{ng} / \mathrm{mL}$ of IL- 6 should be the optimal dosage as it would not affect cell viability (Figure S2C) and it resulted in significantly increased STAT3 transcriptional activity (Figure S2D) as revealed by MTT and luciferase reporter assays respectively. The treatment of $10 \mathrm{ng} / \mathrm{mL}$ of IL-6 could induce tamoxifen resistance in MCF-7 and ZR-75 (Figure 2F,G). Subsequently, we employed STAT3 inhibitor S3I-201 to suppress STAT3 transcriptional activity. One micrometre of S3I-201 was the highest non-lethal concentration (Figure S2E). As expected, the additional of S3I-201 could abolish the effect of IL-6-induced tamoxifen resistance (Figure 2H).

\subsection{Overexpression BQ Could Enhance the Transcription of IL-6 Mediated by Estrogen Receptor $\alpha(E R)$}

From our promoter analysis, we found seven potential ER $\alpha$ binding sites (estrogen response element; ERE) in the promoter region of IL-6, with two of them $(-120$ and +2) close to the transcription initiation site (Figure S3A). We therefore hypothesized that ER $\alpha$ in breast cancer cells could mediate the expression of IL-6. We treated MCF-7 and ZR-75 cells with $1 \mathrm{nM}$ of Estrogen $\left(\mathrm{E}_{2}\right)$. Results from qPCR showed that while $\mathrm{E}_{2}$ treatment suppressed IL-6 expression, its effect was opposite when BQ was overexpressed (Figure 3A). ChIP assay was performed on MCF-7 and ZR-75 to determine whether $1 \mathrm{nM}$ of $\mathrm{E}_{2}$ would affect the binding of $E R \alpha$ to region 1 and region 2 of the IL-6 promoter. The results showed that treatment of $E_{2}$ could only enhance the binding of $E R \alpha$ to region 1 (Figure 3B) but not to region 2 (Figure $3 \mathrm{C}$ ). ChIP assay also showed that overexpression of BQ could further enhance the interaction between $E R \alpha$ and region 1 in the presence of $E_{2}$ (Figure 3D). Furthermore, the expression of IL-6 in the BQ overexpressing cells could be suppressed by gradually increasing the expression of NCOR2 (Figure 3E). Our results suggested that BQ overexpression could enhance the expression of IL-6 through the activity of ER $\alpha$. Together with our previous study [25], we therefore identified a novel molecular pathway to modulate IL-6 expression in ER+ breast cancer (Figure 3F). 


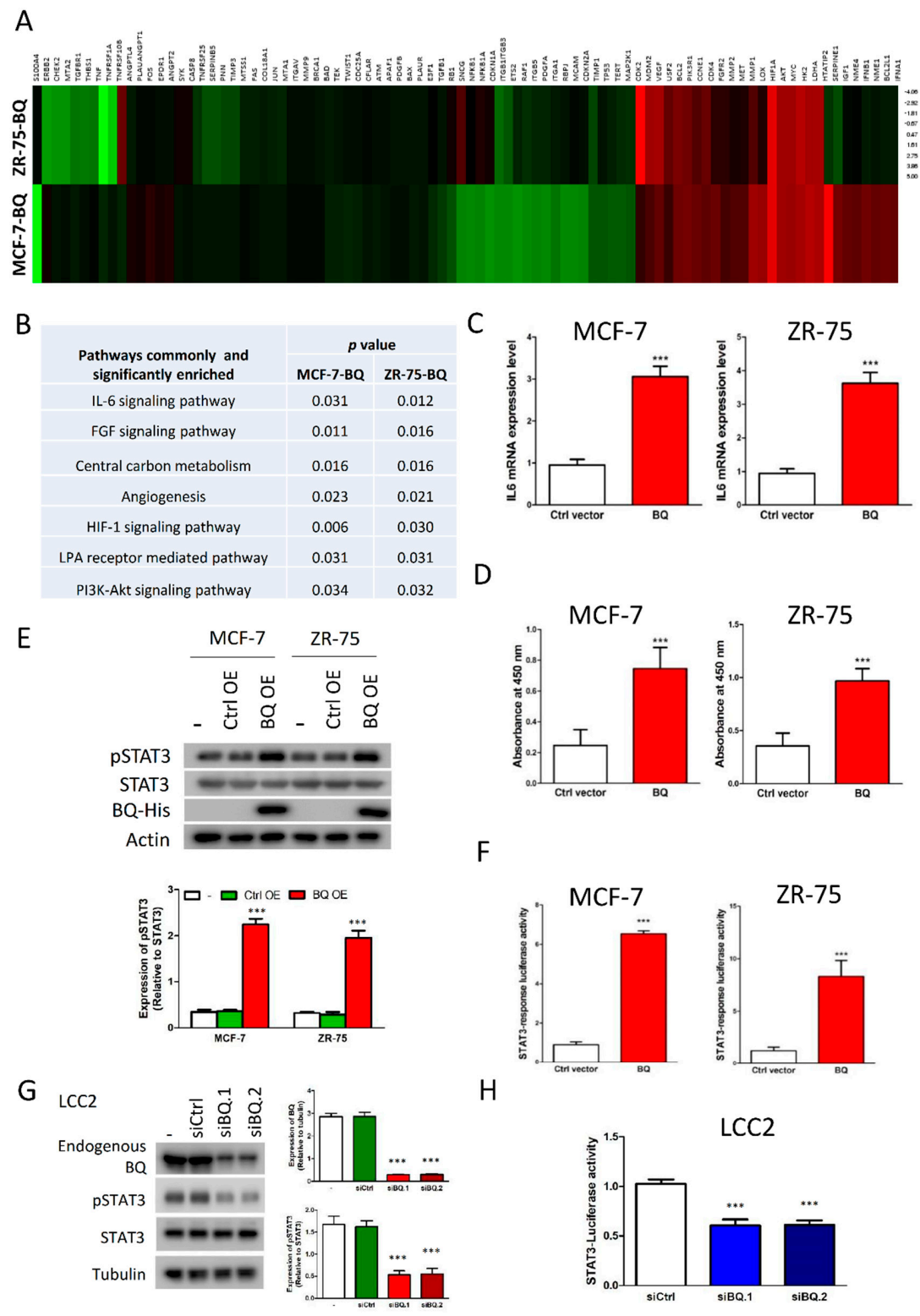

Figure 1. Overexpression of BQ could enhance IL-6/STAT3 signalling pathway in breast cancer cells. (A) The Heatmap showed the expression profiles of 84 cancer-related genes. The comparison was made between BQ overexpressing cells and control cells. Gene expression was compared between BQ overexpressing cells (MCF-7-BQ and ZR-75-BQ) and control cells (MCF-7 and ZR-75). Relative expression was determined and the value of expression data was subjected to $\log _{2}$ transformation for plotting the Heatmap. (B) Signaling pathways commonly and significantly enriched $(p<0.05)$ in the two BQ overexpressing breast cancer cell lines. KEGG pathway enrichment analysis was performed to identify the enriched pathways. (C) Overexpression of BQ could enhance mRNA expression of IL-6. MCF-7 and ZR-75 were transfected with $0.5 \mu \mathrm{g}$ of $p c D N A 3.1 \_B Q$ or $p c D N A 3.1$. qPCR was performed $72 \mathrm{~h}$ post transfection. Untransfected cells were used as reference. Actin was used as internal control. (D) Overexpression of BQ could enhance the production of IL-6. ELISA was performed on the culture medium to detect the level of IL-6. (E) Overexpression of BQ could enhance the expression level of phosphorylated STAT3 (pSTAT3). Western blot was performed to detect the indicated proteins. (F) Overexpression of BQ could enhance the transcriptional activity of STAT3. Luciferase reporter assay was performed $72 \mathrm{~h}$ post transfection to determine STAT3 activity. (G) Knockdown efficiency of siRNA against BQ. LCC2 cells were treated with two independent siRNAs. 20 pmol of the siRNA was used. Western blot was performed $72 \mathrm{~h}$ post transfection. Tubulin was used as loading control. (H) Knockdown of BQ could suppress STAT3 activity in LCC2 cells. Luciferase reporter assay was performed $72 \mathrm{~h}$ post-transfection to determine STAT3 activity. Results were shown as mean \pm SD from at least three independent experiments. Student's $t$ test was used to determine the statistical significance between two groups. ${ }^{* *}$ represents $p<0.001$. 
A

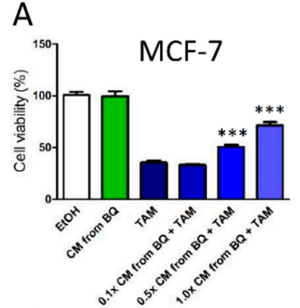

ZR-75

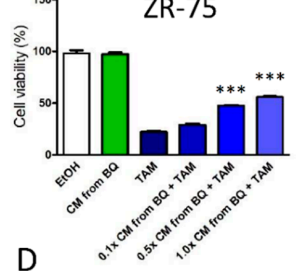

MCF-7

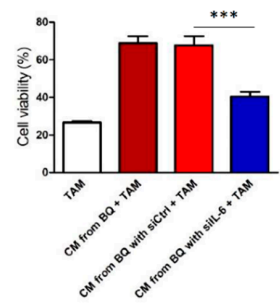

ZR-75

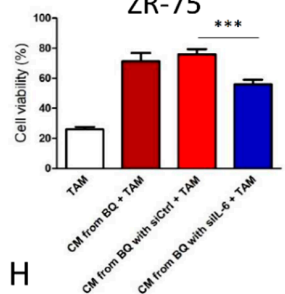

MCF-7

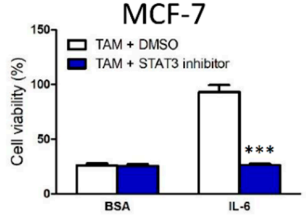

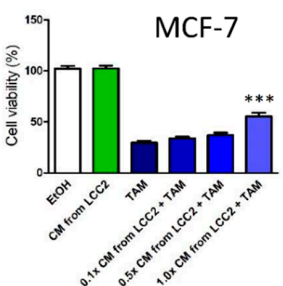

ZR-75
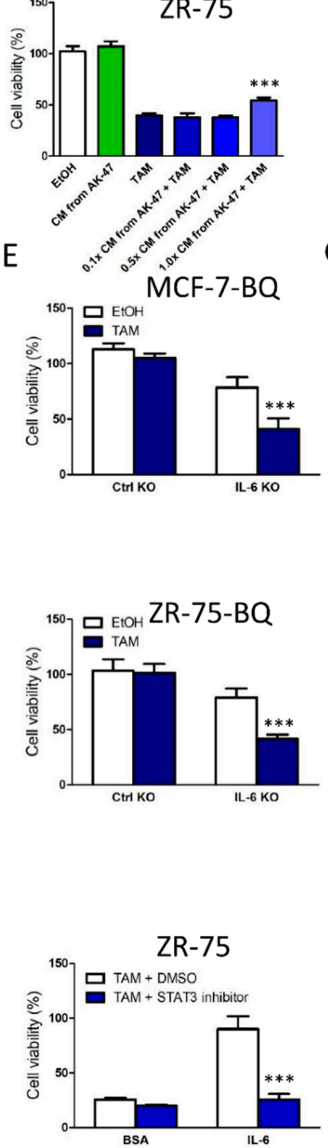

C

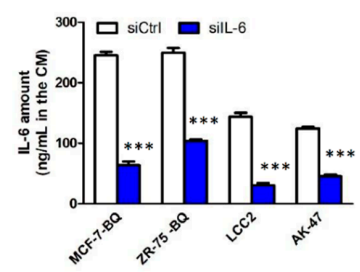

$\mathrm{F}$

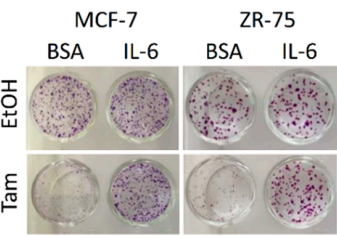

G
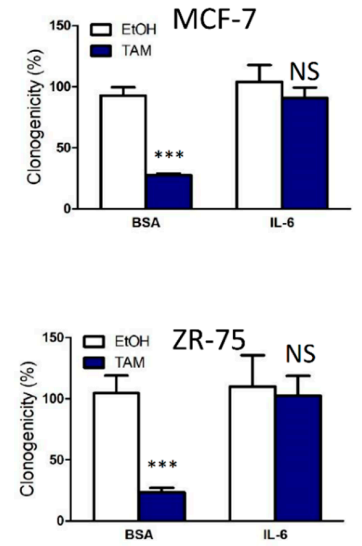

Figure 2. IL-6/STAT3 signalling pathway could modulate tamoxifen response in ER+ breast cancer cells. (A) Conditional medium (CM) from ectopic BQ overexpressing cells could induce tamoxifen resistance. MCF-7 and ZR-75 were cultured in CM obtained from MCF-7-BQ and ZR-75-BQ. The original CM was diluted at $0.1 \times$ and $0.5 \times$. MCF-7 and ZR-75 cells were treated with $5 \mu \mathrm{M}$ of tamoxifen (TAM) for $96 \mathrm{~h}$. MTT assay was employed to determine cell viability. (B) Conditional medium (CM) from high BQ-expressing cells could induce tamoxifen resistance. MCF-7 and ZR-75 were cultured in CM obtained from LCC2 and AK-47. The original CM was diluted at $0.1 \times$ and $0.5 \times$. MCF-7 and ZR-75 cells were treated with $5 \mu \mathrm{M}$ of TAM for $96 \mathrm{~h}$. MTT assay was performed. (C) Treatment of siRNA against IL-6 could reduce the production of IL-6. The cells were treated with the siRNA against IL-6 (siIL-6) or non-targeting siRNA (siCtrl) for $72 \mathrm{~h}$ and ELISA was performed on the cell lysates to detect the amount of IL-6. (D) CM from silL-6-treated cells lost the ability to induce tamoxifen resistance. MCF-7 and ZR-75 were cultured in CM from MCF-7-BQ and ZR-75-BQ, which were treated with siCtrl or silL-6 for $72 \mathrm{~h}$. MCF-7 and ZR-75 cells were treated with $5 \mu \mathrm{M}$ of TAM for $96 \mathrm{~h}$. MTT assay was performed. (E) Knockout of IL-6 could reduce cell viability of BQ-overexpressing cells and resume tamoxifen sensitivity. IL-6 gene was stably knocked out in MCF-7-BQ and ZR-75-BQ. Plasmid-expressing non-targeting gRNA was used as the control. The cells were treated with $5 \mu \mathrm{M}$ of TAM for $96 \mathrm{~h}$. MTT assay was performed. (F) IL-6 could confer tamoxifen resistance. MCF-7 and ZR-75 cells were treated with $10 \mathrm{ng} / \mathrm{mL}$ of recombinant IL- 6 and $5 \mu \mathrm{M}$ of TAM for 2 weeks. Colony formation assay was performed to determine cell viability. (G) Statistical analysis of (F). (H) Inhibition of STAT3 could abolish the effect of IL-6 on tamoxifen resistance. One micrometre of STAT3 inhibitor S3I-201, $10 \mathrm{ng} / \mathrm{mL}$ of recombinant IL-6, and $5 \mu \mathrm{M}$ of TAM were used. MTT assay was performed after $96 \mathrm{~h}$ of the treatment. Results were shown as mean $\pm \mathrm{SD}$ from at least three independent experiments. Students' $t$ test was used to determine the statistical significance between two groups. ${ }^{* * *}$ represents $p<0.001$; NS represents no statistical significance. 
A

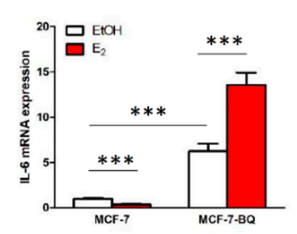

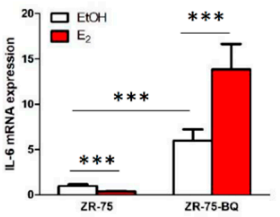

D

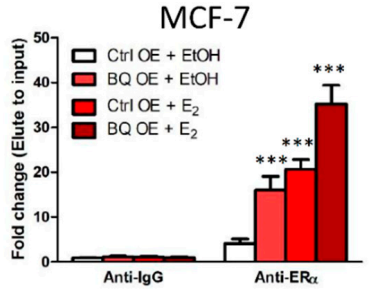

ZR-75

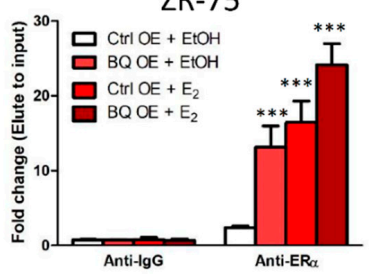

F

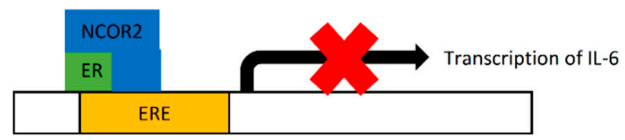

NCO
B

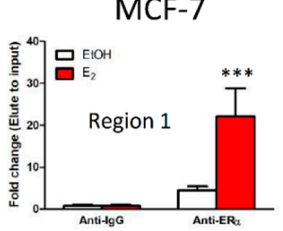

ZR-75

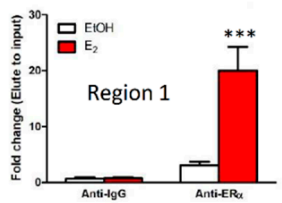

E

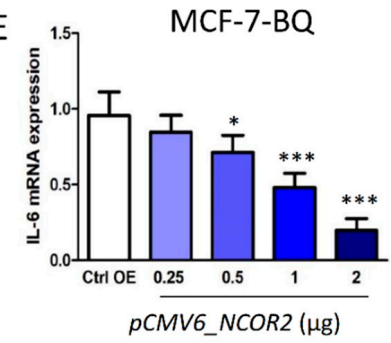

ZR-75-BO

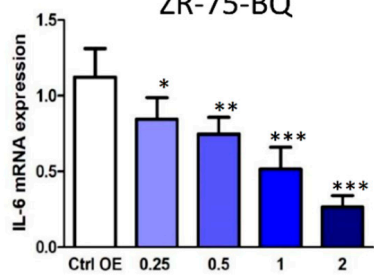

pCMV6_NCOR2 ( $\mu \mathrm{g})$

MCF-7

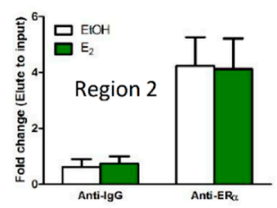

ZR-75

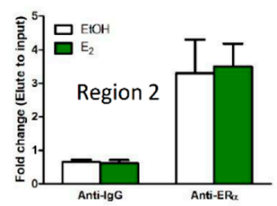

MCF-7-BQ 


\subsection{Overexpression $B Q$ Could Enhance the Transcription of IL-6R Mediated by NF- $k B$}

In order for IL-6/STAT3 signalling to be activated, IL-6 receptor (IL-6R) must be present. We hypothesized that $B Q$ overexpression might enhance the expression of IL-6R in breast cancer and was able to confirm that $\mathrm{BQ}$ overexpression could indeed enhance the expression of IL-6R in breast cancer cells at both mRNA (Figure 4A) and protein (Figure 4B) levels. To address the molecular mechanism through which BQ could enhance the expression of IL-6R, we analysed the promoter region of IL-6R and found the p50 (NF-kB1) binding site to be close to the transcription initiator (Figure S3B). ChIP assay confirmed that p50 could indeed interact with the p50 binding element in the IL-6R promoter region (Figure $4 \mathrm{C}$ ). Since p50 is known to interact with NCOR2 [23], this led us to postulate that $\mathrm{BQ}$, a splice variant of NCOR2, might modulate NF- $\mathrm{KB}$ activity through competition with NCOR2. We first confirmed that NCOR2 could form a protein complex with p50-p65 in breast cancer cells as shown by co-immunoprecipitation (Figure 4D) and next demonstrated that $\mathrm{BQ}$ could compromise the interaction between NCOR2 and p50-p65 (Figure 4E). These results are supportive that BQ can interfere with the repressive effect of NCOR2 on NF- $\mathrm{KB}$ and suggest that BQ overexpression might modulate IL-6R expression by compromising the repressive activity of NCOR2 [23]. To further validate the importance of NF- $\mathrm{kB}$ for IL-6R expression, we examined the effect of NF- $\mathrm{kB}$ inhibitor pXSC, which can covalently modify p50 to abolish its DNA-binding ability. Having first determined that $200 \mathrm{nM}$ of the inhibitor will not affect cell viability of both MCF-7 and ZR-75 (Figure S3C), we demonstrated by qPCR that treatment of the NF- $k B$ inhibitor could indeed suppress IL-6R expression in the BQ-overexpressing cells (Figure 4F). These findings confirm that $B Q$ requires NF- $\mathrm{KB}$ to modulate the expression of IL-6R in breast cancer (Figure 4G).

\subsection{Targeting IL-6R Could Reduce Tamoxifen Resistance}

We next determined the effect of IL-6R knockdown (Figure S4A) on tamoxifen resistance in BQ-overexpressing cells. The results from tamoxifen response assay showed that whilst knockdown of IL-6R itself did not alter tamoxifen response of tamoxifen-sensitive MCF-7 and ZR-75 cells, IL-6R knockdown could reverse the tamoxifen resistance of BQoverexpressing cells to make them sensitive to tamoxifen (Figure 5A). As expected, IL-6R knockdown could compromise the effect of IL-6 on STAT3 activity (Figure S4B). These results suggest that through targeting IL-6R, it may be possible to inhibit IL-6/STAT3 signalling and thus reduce tamoxifen resistance in breast cancer. To address this issue, we employed TCZ, a monoclonal antibody, to bind to IL-6R. We established TCZ of $250 \mathrm{ng} / \mathrm{mL}$ as the maximum non-lethal concentration (Figure $\mathrm{S4C}$ ) and found that the addition of TCZ could reverse tamoxifen resistance in MCF-7-BQ and ZR-75-BQ cells as revealed by MTT (Figure 5B) and clonogenic assay (Figure 5C,D). In addition, we examined whether TCZ could reverse tamoxifen resistance in an animal model. We first generated xenograft models established from ZR-75-BQ and treated the mice with $1 \mathrm{mg} / \mathrm{Kg}$ and $2 \mathrm{mg} / \mathrm{Kg}$ of TCZ together with tamoxifen for 2 months. The results showed that the addition of TCZ could make the tumours become sensitive to tamoxifen in a dose-dependent manner (Figure S5). Next, we determined the effect of TCZ on the xenograft models established from tamoxifen-resistant cell line LCC2, which had a high expression of endogenous BQ. We treated the mice with $2 \mathrm{mg} / \mathrm{Kg}$ of TCZ for 2 months. The results showed that the addition of TCZ could reverse tamoxifen resistance (Figure 5E,F). Such an effect was IL-6Rdependent, as knockdown of IL-6R could abolish the effect of TCZ on tamoxifen response in vivo. Based on our study, we confirmed that targeting IL-6R should be a possible strategy to reverse tamoxifen resistance in breast cancer. 


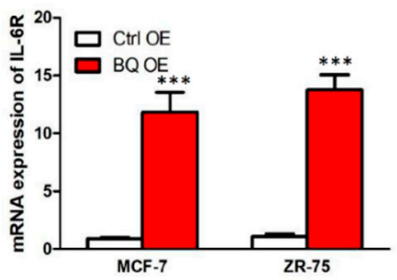

C

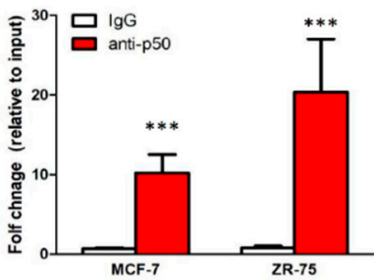

$E$

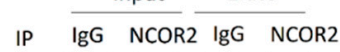

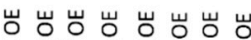

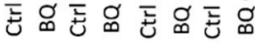
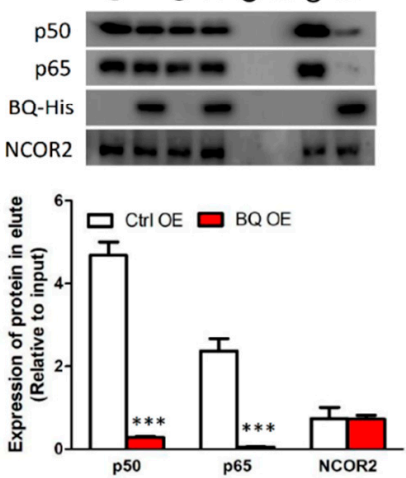

B

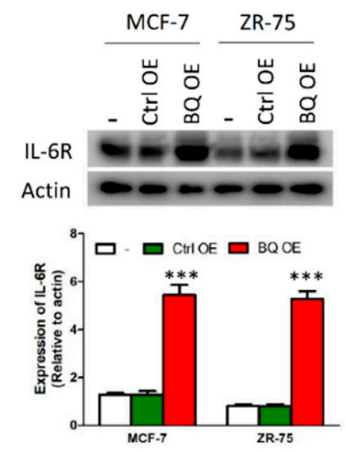

D

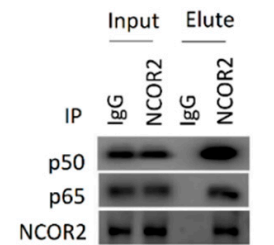

$\mathrm{F}$

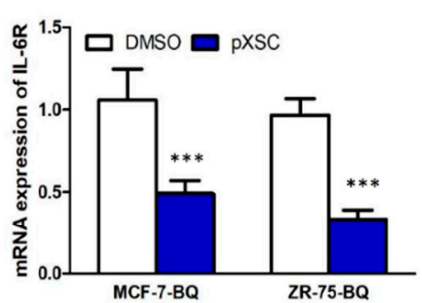

G
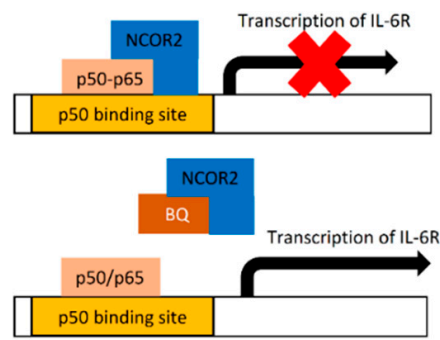

Figure 4. Overexpression of $B Q$ could modulate the expression of IL-6 receptor (IL-6R) in breast cancer. Overexpression of BQ could enhance (A) mRNA and (B) protein levels of IL-6R. qPCR was performed to determine IL-6R expression in stable BQ-overexpressing cells and their control cells. Parental MCF-7 and ZR-75 were used as reference. Western blot was employed to determine IL-6R. Actin was used as loading control. (C) p50 could bind to the promoter of IL-6R. ChIP assay was performed with anti-p50. qPCR was used to detect the relative amount of DNA with the promoter of IL-6R. (D) NCOR2 could interact with p50 and p65. p50 and p65 are two subunits of NF-kB. Co-immunoprecipitation was performed on MCF-7. Anti-NCOR2 was used to immunoprecipitate the protein complex. Western blot was performed to determine the presence of the indicated protein candidates. (E) Overexpression of BQ could compromise the interaction between NCOR2 and NF-kB. Co-immunoprecipitation was performed on MCF-7 with anti-NCOR2. Western blot was employed to determine the presence of the indicated proteins in the immunoprecipitant. (F) Inhibition of NF-kB could suppress the expression of IL-6R in BQ overexpressing cells. Two hundred nanomolar of NF-kB inhibitor pXSC was used. The cells were treated for $72 \mathrm{~h}$. qPCR was performed to determine IL-6R expression. Actin was used as internal control. (G) Schematic diagram showing the effect of $B Q$ overexpression on the modulation of IL-6R expression through the NF-kB-dependent pathway. In the presence of $\mathrm{BQ}$ overexpression, $\mathrm{BQ}$ binds with NCOR2. This interaction compromises the ability of NCOR2 to bind to NF-kB to repress transcription mediated by NF-kB. Results were shown as mean \pm SD from at least three independent experiments. Students' $t$ test was used to determine the statistical significance between two groups. ${ }^{* * *}$ represents $p<0.001$. 


\subsection{Clinical Significance of $B Q$ and IL-6R in Breast Cancer}

To provide further in vivo evidence to support our findings, we correlated the expression of BQ and IL-6R in clinical breast cancer samples. Immunohistochemistry (IHC) was performed on TMA of primary breast cancer cases to examine the expression of $B Q$ and IL-6R in breast cancer tissues. BQ expression is functional in the nucleus, hence nuclear expression was assessed by H-score, whilst for IL-6R expression, being localized to the cytoplasm, the cytoplasmic score was used. The median values of both scores were used to dichotomize into low and high expression groups (Figure 6A). Correlating BQ nuclear with IL-6R cytoplasmic expressions in ER+ breast cancer, we found statistically significant direct correlation between IL-6R and BQ expression ( $p=0.027$ Mann-Whitney $\mathrm{U}$ test), as well as positive correlation by chi-square test $(p=0.011)$ (Figure 6B). Moreover, high expression of IL-6R was significantly correlated with tamoxifen resistance in these ER+ breast cancer cases ( $p=0.005$ Mann-Whitney $\mathrm{U}$ test), as well as by chi-square test $\left(p=1.9 \times 10^{-5}\right)$ (Figure 6C). This was also demonstrated by Kaplan-Meier survival analysis showing patients with high expression of IL-6R with poorer outcome for both overall survival ( $p=0.002$; Figure $6 \mathrm{D})$ and disease-specific survival $(p=0.005$; Figure $6 \mathrm{E})$. By univariate cox regression analysis, high expression of IL-6R was significantly associated with poorer overall survival ( $p=0.004$; RR: $3.71695 \%$ CI: $1.537,8.984$; Table 3 ) as well as poorer disease-specific survival ( $p=0.008$; RR: 5.664 95\% CI: 1.569, 20.441; Table 4). Multivariate cox-regression analyses showed that after adjustment for the other variables, high expression of IL-6R remained significantly associated with poorer overall survival as well as disease-specific survival ( $p=0.036$; RR: 10.967 95\% CI: 1.169, 102.878; Table 3); $(p=0.009$; RR: 5.586 95\% CI: 1.534, 20.349; Table 4) respectively. Altogether, our results confirmed high expression of IL-6R in ER+ breast cancer was associated with tamoxifen resistance and poorer survival outcome; and IL-6R could be an independent prognostic factor.

Table 3. Cox regression analyses of overall survival in ER+ breast cancer patients.

\begin{tabular}{|c|c|c|c|c|}
\hline \multirow{2}{*}{$\frac{\text { Clinical-Pathological Parameters }}{\text { Clinical characteristic }}$} & \multicolumn{2}{|c|}{ Univariate Analysis } & \multicolumn{2}{|c|}{ Multivariate Analysis } \\
\hline & RR $(95 \%$ CI) & $p$ Value & $\mathrm{RR}(95 \% \mathrm{CI})$ & $p$ Value \\
\hline Age $(n=69)$ & $1.975(0.931,4.188)$ & 0.076 & & \\
\hline T-stage $(n=28)$ & $8.097(1.441,45.491)$ & 0.018 & $4.262(0.561,32.405)$ & 0.161 \\
\hline Lymph-node involvement $(n=63)$ & $0.904(0.412,1.986)$ & 0.802 & & \\
\hline Tumor-Grade $(n=68)$ & $1.171(0.550,2.497)$ & 0.682 & & \\
\hline Histological type $(n=69)$ & $1.166(0.351,3.873)$ & 0.802 & & \\
\hline HER2 status $(n=48)$ & $1.159(0.445,3.016)$ & 0.762 & & \\
\hline Tumor size $(n=52)$ & $0.941(0.388,2.278)$ & 0.892 & & \\
\hline Cases with high IL-6R cytoplasm score $(n=55)$ & $3.716(1.537,8.984)$ & 0.004 & $10.967(1.169,102.878)$ & 0.036 \\
\hline
\end{tabular}

Table 4. Cox regression analyses of disease specific survival in ER+ breast cancer patients.

\begin{tabular}{|c|c|c|c|c|}
\hline \multirow{2}{*}{$\begin{array}{c}\text { Clinical-Pathological Parameters } \\
\text { Clinical characteristic }\end{array}$} & \multicolumn{2}{|c|}{ Univariate Analysis } & \multicolumn{2}{|c|}{ Multivariate Analysis } \\
\hline & RR $(95 \%$ CI $)$ & $p$ Value & $\mathrm{RR}(95 \% \mathrm{CI})$ & $p$ Value \\
\hline Age $(n=69)$ & $1.198(0.472,3.040)$ & 0.703 & & \\
\hline T-stage $(n=28)$ & $8.097(1.441,45.491)$ & 0.018 & & \\
\hline Lymph-node involvement $(n=63)$ & $1.402(0.508,3.864)$ & 0.514 & & \\
\hline Tumor-Grade $(n=68)$ & $3.672(1.208,11.162)$ & 0.022 & $4.612(1.298,16.386)$ & 0.018 \\
\hline Histological type $(n=71)$ & $1.022(0.235,4.452)$ & 0.976 & & \\
\hline HER2 status $(n=48)$ & $1.777(0.517,6.110)$ & 0.361 & & \\
\hline Tumor size $(n=52)$ & $1.472(0.442,4.898)$ & 0.529 & & \\
\hline Cases with high IL-6R cytoplasm score $(n=55)$ & $5.664(1.569,20.441)$ & 0.008 & $5.586(1.534,20.349)$ & 0.009 \\
\hline
\end{tabular}


A

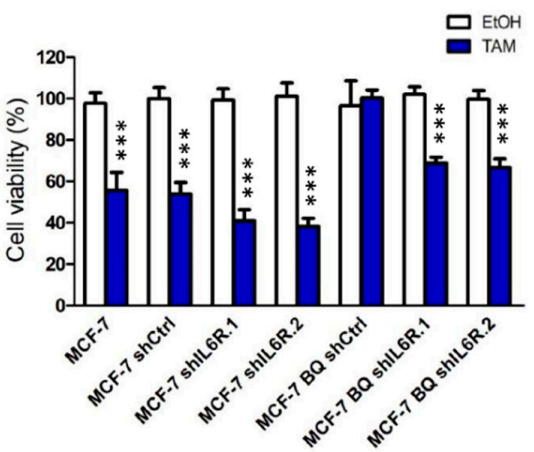

B

MCF-7-BO
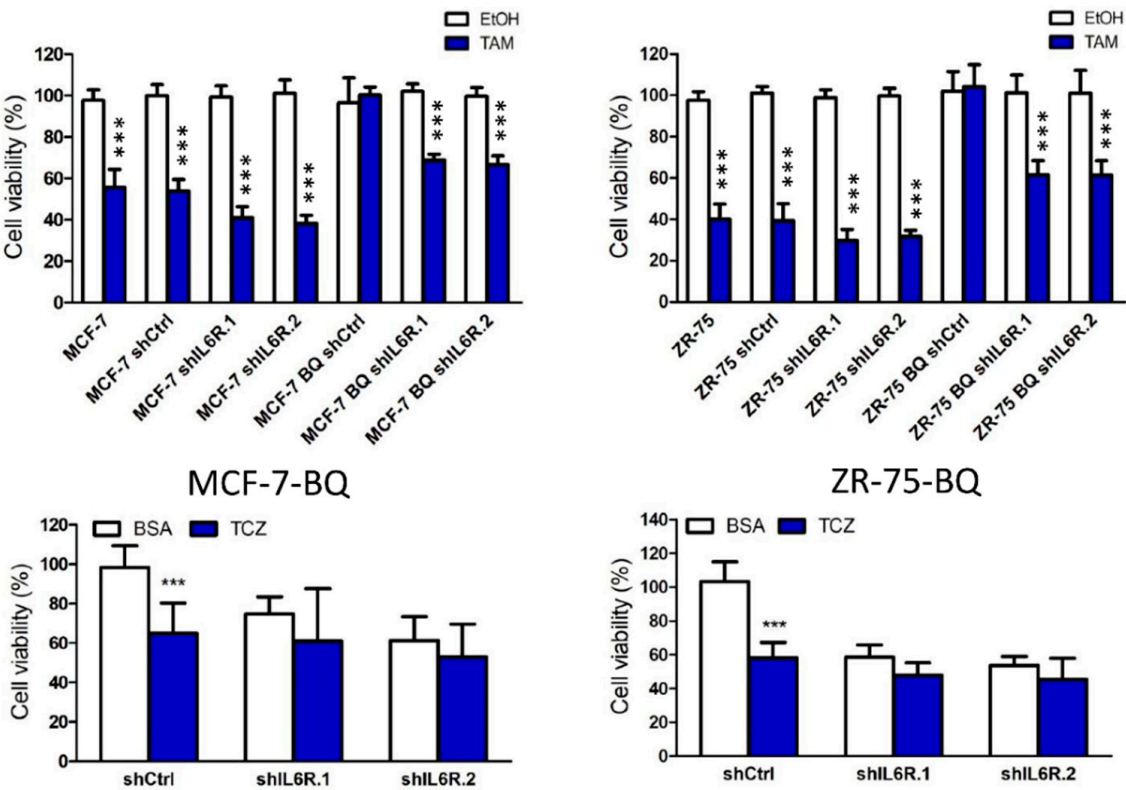

C

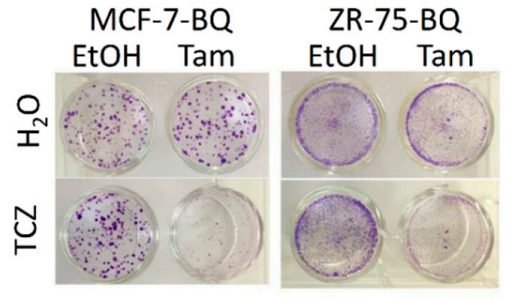

D

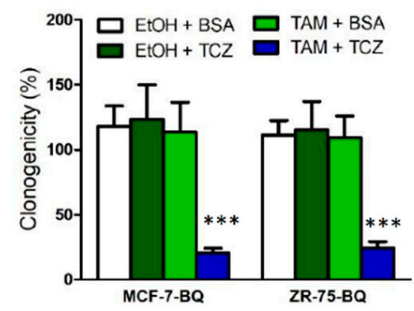

E

$\mathrm{F}$
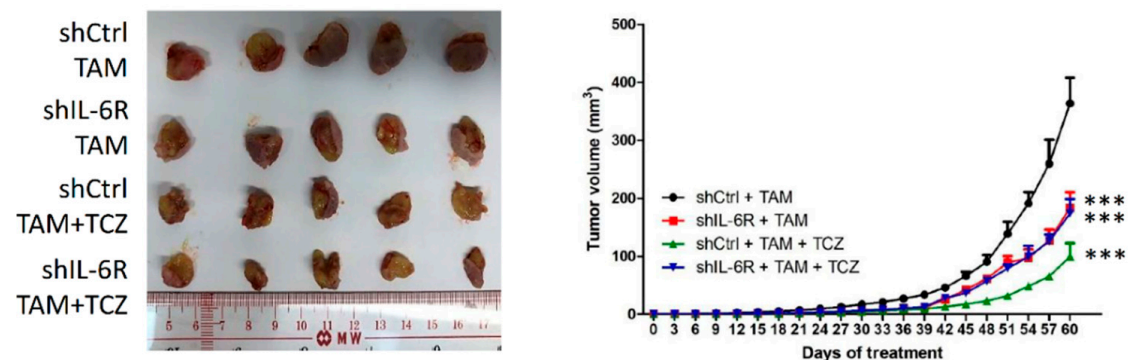

Figure 5. Targeting IL-6R could reverse tamoxifen (TAM) resistance in BQ overexpressing ER+ breast cancer. (A) Knockdown of IL-6R could enhance the efficacy of TAM in control cells and reverse TAM resistance in BQ overexpressing cells. Cell lines with stable IL-6R knockdown were used. The cells were treated with $5 \mu \mathrm{M}$ of TAM for $96 \mathrm{~h}$. MTT was used to determine cell viability. (B) Tocilizumab (TCZ) could reduce cell viability in BQ overexpressing cells in the presence of $5 \mu \mathrm{M}$ of TAM. The effect of TCZ on cell viability was compromised by IL-6R knockdown. Two-hundred-and-fifty $\mathrm{ng} / \mathrm{mL}$ of TCZ or BSA was used. MTT assay was performed after $96 \mathrm{~h}$ of the treatment. (C) TCZ could reverse TAM resistance in BQ-overexpressing cells. The cells were treated with $250 \mathrm{ng} / \mathrm{mL}$ of TCZ and $5 \mu \mathrm{M}$ of TAM for 2 weeks. Clonogenic assay was performed. (D) Statistical analysis of (C). (E) TCZ could reduce TAM resistant in vivo. TAM-resistance cell lines LCC2 shCtrl and LCC2 shIL-6R (shIL-6R.1) were used for xenograft establishment. The mice were randomized into different groups. The mice received $0.5 \mathrm{mg}$ of TAM and $2 \mathrm{mg} / \mathrm{Kg}$ of TCZ through subcutaneous injection. The mice were treated twice per week for 8 weeks. (F) The graph showed the volume change of tumors during the treatment period. Results were shown as mean \pm SD from at least three independent experiments. Students' $t$ test was used to determine the statistical significance between treatment and control groups. ${ }^{* * *}$ represents $p<0.001$. 
A
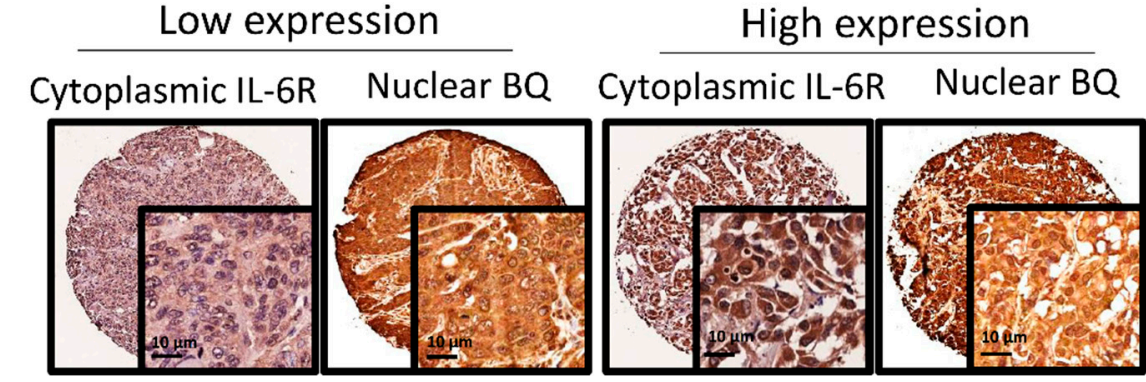

B
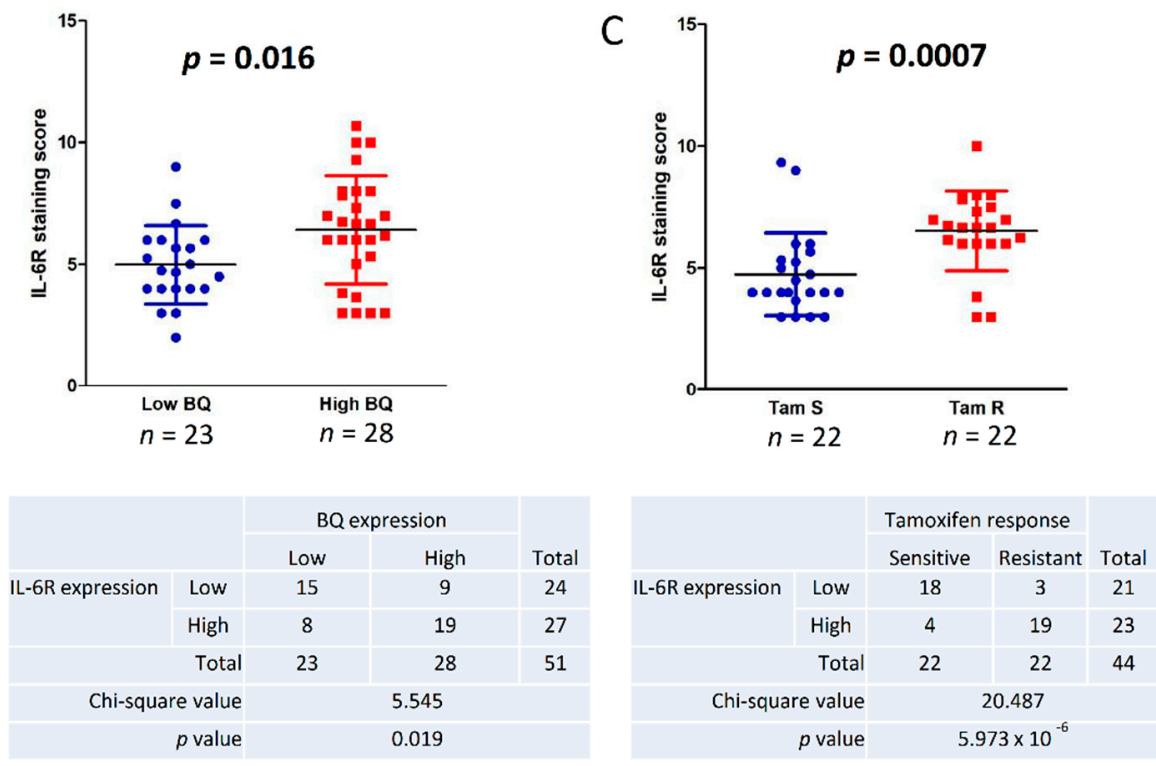

D

$E$
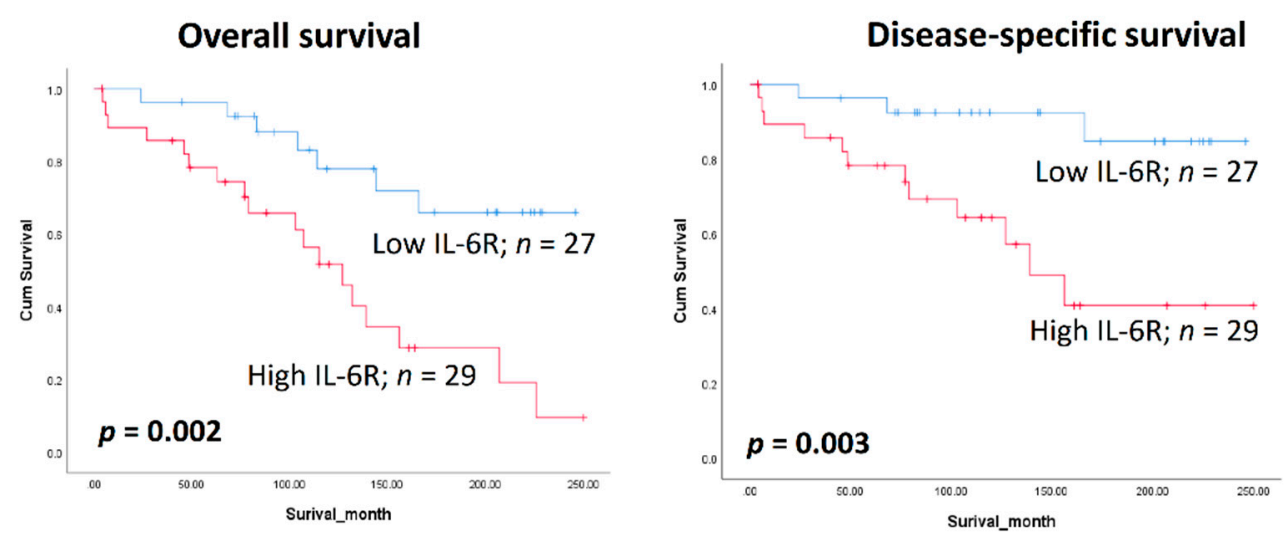

Figure 6. Clinical significance of IL-6R in ER+ breast cancer. (A) IHC showing representative high and low expressions of cytoplasmic IL-6R and nuclear BQ respectively. (B) Expression of IL-6R was compared with low nuclear BQ and high nuclear BQ expression groups. Mann-Whitney $U$ test was employed to determine the statistical significance between two groups. Chi-square text was used to determine the correlation between the expression of IL-6R and BQ. (C) Expression of IL-6R was significantly high ( $p=0.005$; Mann-Whitney U test; $p=1.9 \times 10^{-5}$; Chi-square test). Kaplan-Meier analysis showing (D) overall survival ( $p=0.002$; log-rank test) and (E) disease-specific survival ( $p=0.003$; log-rank test). Patients with high expression of IL-6R $(n=29)$ have a poorer survival outcome compared with patients with low IL-6R expression $(n=27)$.

\section{Discussion}

Our study illustrates that overexpression of $B Q$ can modulate tamoxifen resistance by enhancing the expression of IL-6 and IL-6R in ER+ breast cancer. Through molecular studies, we illustrated that $\mathrm{BQ}$ through interacting with NCOR2 could interfere with its 
function. NCOR2 itself is a central component for mediating gene repression [28]. NCOR2 functions as a dimer in the corepressor complex, recruiting other corepressor proteins such as GPS, TBLR1, and HDAC3 to mediate the repression of transcription factor activity to suppress gene expression. Our previous study identified $B Q$, a novel splice variant of NCOR2, which retains the N-terminus of NCOR [24]. We showed that BQ could interact with NCOR2 and this interaction could disrupt the interaction between NCOR2 with other regulatory factors, thus targeting transcription factors such as ER $\alpha$ [25]. In the current study, we identified two potential ER $\alpha$ binding sites within the promoter region of IL-6 (Figure S3A). Previous studies have shown that the addition of estrogen could impair the expression of IL-6/STAT3 signalling pathway through ER and STAT3 modulator PIAS $[29,30]$. One of these studies found that activation of ER could occupy the NF- $k B$ binding site in the promoter of IL-6, thus blocking the transcription of IL-6.

In our study, we observed that overexpression of BQ reversed the effect of ER on IL-6 expression as demonstrated by the enhanced IL-6 expression shown in Figure 3A. In the presence of BQ overexpression, ER could bind to ERE in the IL-6 promoter and the addition of estrogen could further enhance the proportion of ER binding to this region (Figure 3D). We believe this enhanced binding in turn enhanced the expression of IL-6. One possible reason could be the interaction between BQ and NCOR2. NCOR2 is a repressor of gene expression; and the interaction between NCOR2 and ER is believed to suppress the initiation of transcription [22]. BQ overexpression, in interacting and competing with NCOR2, would diminish the suppressive role of NCOR2 on IL-6 expression in the presence of ER.

Interleukin-6 (IL-6) is a multipotent cytokine that plays an important role in immune responses and human diseases, including different type of cancers [31]. A high concentration of serum IL-6 has been shown to be associated with aggressive tumour types and poor disease-free and overall survival [14]. IL-6 binds to IL-6 receptor (IL-6R) to activate STAT3. Constitutive activation of STAT3 has frequently been observed in a variety of tumours, including breast cancer [32], and such activation can promote proliferation and survival of cancer cells [33]. Recent reports showed that STAT3 activation is associated with drug resistance outcomes, and that blockade of STAT3 pathway can restore the efficacy of chemodrugs. Through activation of the STAT3/SNAIL pathway, cisplatin treatment rendered drug resistance, enhanced the EMT-like phenotype, and increased migration and invasion abilities in tumour cells [34]. In addition, STAT3 activation also mediates resistance to chemotherapeutic agents 5-fluorouracil (5-FU), oxaliplatin, and SN-380 [35], suggesting that STAT3 activation is responsible for such resistance. In breast cancer, STAT3 activation is associated with tamoxifen resistance [36]. Therefore, inhibition of STAT3 signaling will be an attractive strategy to combat drug resistance.

In our study, we confirmed that overexpression of BQ could lead to the activation of IL-6/STAT3 pathway (Figure 1), which conferred resistance to tamoxifen in ER+ breast cancer cells (Figure 2). The activation process required the presence of ER $\alpha$ and NF-kB that modulated the expression of IL-6 (Figure 3) and IL-6R (Figure 4) respectively. Instead of direct inhibition of STAT3 via any small inhibitor, we proposed to suppress STAT3 activity by compromising its activation process. We showed that Tocilizumab (TCZ), an FDAapproved anti-IL-6R antibody for the treatment of patients with rheumatoid arthritis, could reverse tamoxifen resistance in vitro and in vivo (Figure 5). We have successfully illustrated that targeting IL-6R could be an alternative way to suppress IL-6/STAT3 pathway in breast cancer and demonstrate the usage of TCZ could reduce tamoxifen resistance in breast cancer.

To assess the usefulness of TCZ in overcoming tamoxifen resistance, we first ascertained the expression of IL-6R in breast cancer tissue. The protein expression of IL-6R was determined by IHC on TMA of primary breast cancer (Figure 6A), and the expression level of IL-6R was shown to be significantly correlated with tamoxifen resistance (Figure 6C). In vitro study demonstrated that BQ could modulate the expression of IL-6R (Figure 4), while in vivo we found a positive correlation between BQ and IL-6R (Figure 6B). Moreover, 
high expression of IL-6R showed significant association with poorer prognosis as indicated both by Kaplan-Meier (Figure $6 \mathrm{D}-\mathrm{G}$ ) and cox regression (Table 2) survival analyses. These results support the clinical significance of IL-6R in breast cancer. Thus, our study not only confirms that IL-6R could be an independent prognostic factor in ER+ breast cancer, but could also be a possible target to suppress tamoxifen resistance.

\section{Conclusions}

Our study has elucidated the molecular mechanism through which BQ can modulate the expression of IL- 6 and IL-6R and thus the activation of STAT3 in breast cancer. This mechanism could contribute to the development of tamoxifen resistance in breast cancer. We have shown that targeting the IL-6/STAT3 signalling pathway by TCZ could successfully reverse tamoxifen resistance in vitro and in vivo. Our results highlight the significance of IL-6R in ER+ breast cancer and provides the basis for the development of a novel strategy for reversing tamoxifen resistance in breast cancer. Since TCZ has approval for clinical use in various immunological inflammatory conditions, it might thus be more easily considered for clinical trial in the management of breast cancer patients.

Supplementary Materials: The following are available online at https:/ /www.mdpi.com/2072-6 694/13/7/1511/s1, Figure S1: Supplementary information to Figure 1, Figure S2: Supplementary information to Figure 2, Figure S3: Supplementary information to Figure 3, Figure S4: Supplementary information to Figure 4, Figure S5: Supplementary information to Figure 5, Figure S6: Uncropped blots used in main figures, Figure S7: Uncropped blots used in supplementary figures, Table S1: The effect of BQ on the expression of candidate genes in the PCR array, Table S2: Affected genes by BQ overexpression in each of the pathways.

Author Contributions: Conceptualization, H.T. and U.-S.K.; Data curation, H.T., E.P.S.M., K.M.C. and U.-S.K.; Formal analysis, H.T. and U.-S.K.; Funding acquisition, H.T. and U.-S.K.; Investigation, H.T. and U.-S.K.; Methodology, H.T., E.P.S.M., K.M.C. and U.-S.K.; Project administration, E.P.S.M. and U.-S.K.; Resources, U.-S.K.; Software, E.P.S.M. and U.-S.K.; Supervision, H.T. and U.-S.K.; Validation, H.T. and E.P.S.M.; Visualization, H.T., E.P.S.M. and U.-S.K.; Writing-original draft, H.T. and U.-S.K.; Writing-review \& editing, H.T. and U.-S.K. All authors have read and agreed to the published version of the manuscript.

Funding: This project was kindly supported by Health and Medical Research Fund, HKSAR (06171696 and 06171916) and the Committee on Research and Conference Grants from the University of Hong Kong Project number 201811159118, 201910160022 and 201910159174.

Institutional Review Board Statement: This study included the use of human samples and clinical information. Ethical approval (UW 08-147) was granted by the Institutional Review Board of The University of Hong Kong for the permission of using human samples in the current study.

Informed Consent Statement: Informed consent was obtained from all subjects involved in the study.

Data Availability Statement: Data is contained within the Supplementary Material (Table S1).

Acknowledgments: We thank L.S.W., W.L.C. and S.Y.C. for their assistance in retrieving clincial followup data of the breast cancer cases in this study.

Conflicts of Interest: The authors declare no conflict of interest.

\section{References}

1. Perou, C.M.; Sorlie, T.; Eisen, M.B.; van de Rijn, M.; Jeffrey, S.S.; Rees, C.A.; Pollack, J.R.; Ross, D.T.; Johnsen, H.; Akslen, L.A.; et al. Molecular portraits of human breast tumours. Nature 2000, 406, 747-752. [CrossRef]

2. Siersbaek, R.; Kumar, S.; Carroll, J.S. Signaling pathways and steroid receptors modulating estrogen receptor a function in breast cancer. Gene Dev. 2018, 32, 1141-1154. [CrossRef]

3. Hu, R.; Hilakivi-Clarke, L.; Clarke, R. Molecular mechanisms of tamoxifen-associated endometrial cancer. Oncol. Lett. 2015, 9 , 1495-1501. [CrossRef]

4. Matutino, A.; Joy, A.A.; Brezden-Masley, C.; Chia, S.; Verma, S. Hormone receptor-positive, HER2-negative metastatic breast cancer: Redrawing the lines. Curr. Oncol. 2018, 25, S131-S141. [CrossRef]

5. Ring, A.; Dowsett, M. Mechanisms of tamoxifen resistance. Endocr. Relat. Cancer 2004, 11, 643-658. [CrossRef] 
6. Shou, J.; Massarweh, S.; Osborne, C.K.; Wakeling, A.E.; Ali, S.; Weiss, H.; Schiff, R. Mechanisms of tamoxifen resistance: Increased estrogen receptor-HER2/neu cross-talk in ER/HER2-positive breast cancer. J. Natl. Cancer Inst. 2004, 96, 926-935. [CrossRef] [PubMed]

7. Riggins, R.B.; Schrecengost, R.S.; Guerrero, M.S.; Bouton, A.H. Pathways to tamoxifen resistance. Cancer Lett. 2007, $256,1-24$. [CrossRef] [PubMed]

8. Sette, C.; Ladomery, M.; Ghigna, C. Alternative splicing: Role in cancer development and progression. Int. J. Cell Biol. 2013, 2013, 421606. [CrossRef]

9. Hunter, C.A.; Jones, S.A. IL-6 as a keystone cytokine in health and disease. Nat. Immunol. 2015, 16, 448-457. [CrossRef] [PubMed]

10. Mauer, J.; Denson, J.L.; Bruning, J.C. Versatile functions for IL-6 in metabolism and cancer. Trends Immunol. 2015, 36, 92-101. [CrossRef]

11. Johnson, D.E.; O'Keefe, R.A.; Grandis, J.R. Targeting the IL-6/JAK/STAT3 signalling axis in cancer. Nat. Rev. Clin. Oncol. 2018, 15, 234-248. [CrossRef]

12. Siersbaek, R.; Scabia, V.; Nagarajan, S.; Chernukhin, I.; Papachristou, E.K.; Broome, R.; Johnston, S.J.; Joosten, S.E.P.; Green, A.R.; Kumar, S.; et al. IL6/STAT3 Signaling Hijacks Estrogen Receptor alpha Enhancers to Drive Breast Cancer Metastasis. Cancer Cell 2020, 38, 412-423.e9. [CrossRef] [PubMed]

13. Jiang, M.; Chen, J.; Zhang, W.; Zhang, R.; Ye, Y.; Liu, P.; Yu, W.; Wei, F.; Ren, X.; Yu, J. Interleukin-6 Trans-Signaling Pathway Promotes Immunosuppressive Myeloid-Derived Suppressor Cells via Suppression of Suppressor of Cytokine Signaling 3 in Breast Cancer. Front. Immunol. 2017, 8, 1840. [CrossRef]

14. Bachelot, T.; Ray-Coquard, I.; Menetrier-Caux, C.; Rastkha, M.; Duc, A.; Blay, J.Y. Prognostic value of serum levels of interleukin 6 and of serum and plasma levels of vascular endothelial growth factor in hormone-refractory metastatic breast cancer patients. Br. J. Cancer 2003, 88, 1721-1726. [CrossRef] [PubMed]

15. Salgado, R.; Junius, S.; Benoy, I.; Van Dam, P.; Vermeulen, P.; Van Marck, E.; Huget, P.; Dirix, L.Y. Circulating interleukin-6 predicts survival in patients with metastatic breast cancer. Int. J. Cancer 2003, 103, 642-646. [CrossRef] [PubMed]

16. Huynh, J.; Chand, A.; Gough, D.; Ernst, M. Therapeutically exploiting STAT3 activity in cancer-Using tissue repair as a road map. Nat. Rev. Cancer 2019, 19, 82-96. [CrossRef]

17. Hashizume, M.; Tan, S.L.; Takano, J.; Ohsawa, K.; Hasada, I.; Hanasaki, A.; Ito, I.; Mihara, M.; Nishida, K. Tocilizumab, a Humanized Anti-IL-6R Antibody, as an Emerging Therapeutic Option for Rheumatoid Arthritis: Molecular and Cellular Mechanistic Insights. Int. Rev. Immunol. 2015, 34, 265-279. [CrossRef]

18. Bobbo, V.C.D.; Jara, C.P.; Mendes, N.F.; Morari, J.; Velloso, L.A.; Araujo, E.P. Interleukin-6 Expression by Hypothalamic Microglia in Multiple Inflammatory Contexts: A Systematic Review. Biomed. Res. Int. 2019, 2019, 1365210. [CrossRef] [PubMed]

19. Oeckinghaus, A.; Ghosh, S. The NF-kappaB family of transcription factors and its regulation. Cold Spring Harb. Perspect. Biol. 2009, 1, a000034. [CrossRef] [PubMed]

20. Giuliani, C.; Bucci, I.; Napolitano, G. The Role of the Transcription Factor Nuclear Factor-kappa B in Thyroid Autoimmunity and Cancer. Front. Endocrinol. 2018, 9, 471. [CrossRef]

21. Battaglia, S.; Maguire, O.; Campbell, M.J. Transcription factor co-repressors in cancer biology: Roles and targeting. Int. J. Cancer 2010, 126, 2511-2519. [CrossRef]

22. Peterson, T.J.; Karmakar, S.; Pace, M.C.; Gao, T.; Smith, C.L. The silencing mediator of retinoic acid and thyroid hormone receptor (SMRT) corepressor is required for full estrogen receptor alpha transcriptional activity. Mol. Cell Biol. 2007, 27, 5933-5948. [CrossRef] [PubMed]

23. Espinosa, L.; Ingles-Esteve, J.; Robert-Moreno, A.; Bigas, A. IkappaBalpha and p65 regulate the cytoplasmic shuttling of nuclear corepressors: Cross-talk between Notch and NFkappaB pathways. Mol. Biol. Cell 2003, 14, 491-502. [CrossRef] [PubMed]

24. Zhang, L.D.; Gong, C.; Lau, S.L.Y.; Yang, N.; Wong, O.G.W.; Cheung, A.N.Y.; Tsang, J.W.H.; Chan, K.Y.K.; Khoo, U.S. SpliceArray Profiling of Breast Cancer Reveals a Novel Variant of NCOR2/SMRT That Is Associated with Tamoxifen Resistance and Control of ER alpha Transcriptional Activity. Cancer Res. 2013, 73, 246-255. [CrossRef] [PubMed]

25. Gong, C.; Man, E.P.S.; Tsoi, H.; Lee, T.K.W.; Lee, P.; Ma, S.T.; Wong, L.S.; Luk, M.Y.; Rakha, E.A.; Green, A.R.; et al. BQ323636.1, a Novel Splice Variant to NCOR2, as a Predictor for Tamoxifen-Resistant Breast Cancer. Clin. Cancer Res. 2018, 24, 3681-3691. [CrossRef] [PubMed]

26. Wong, L.J.; Dai, P.; Lu, J.F.; Lou, M.A.; Clarke, R.; Nazarov, V. AIB1 gene amplification and the instability of polyQ encoding sequence in breast cancer cell lines. BMC Cancer 2006, 6, 111. [CrossRef] [PubMed]

27. Dreos, R.; Ambrosini, G.; Perier, R.C.; Bucher, P. The Eukaryotic Promoter Database: Expansion of EPDnew and new promoter analysis tools. Nucleic Acids Res. 2015, 43, D92-D96. [CrossRef]

28. Mottis, A.; Mouchiroud, L.; Auwerx, J. Emerging roles of the corepressors NCoR1 and SMRT in homeostasis. Genes Dev. 2013, 27, 819-835. [CrossRef]

29. Wang, L.H.; Yang, X.Y.; Mihalic, K.; Xiao, W.H.; Li, D.P.; Farrar, W.L. Activation of estrogen receptor blocks interleukin-6-inducible cell growth of human multiple myeloma involving molecular cross-talk between estrogen receptor and STAT3 mediated by co-regulator PLAS3. J. Biol. Chem. 2001, 276, 31839-31844. [CrossRef]

30. Galien, R.; Garcia, T. Estrogen receptor impairs interleukin-6 expression by preventing protein binding on the NF-kappa B site. Nucleic Acids Res. 1997, 25, 2424-2429. [CrossRef] 
31. Tanaka, T.; Narazaki, M.; Kishimoto, T. IL-6 in Inflammation, Immunity, and Disease. Csh. Perspect. Biol. 2014,6 , a016295. [CrossRef] [PubMed]

32. Yu, H.; Lee, H.; Herrmann, A.; Buettner, R.; Jove, R. Revisiting STAT3 signalling in cancer: New and unexpected biological functions. Nat. Rev. Cancer 2014, 14, 736-746. [CrossRef] [PubMed]

33. Crescenzo, R.; Abate, F.; Lasorsa, E.; Tabbo', F.; Gaudiano, M.; Chiesa, N.; Di Giacomo, F.; Spaccarotella, E.; Barbarossa, L.; Ercole, E.; et al. Convergent Mutations and Kinase Fusions Lead to Oncogenic STAT3 Activation in Anaplastic Large Cell Lymphoma. Cancer Cell 2015, 27, 516-532. [CrossRef]

34. Liu, W.H.; Chen, M.T.; Wang, M.L.; Lee, Y.Y.; Chiou, G.Y.; Chien, C.S.; Huang, P.I.; Chen, Y.W.; Huang, M.C.; Chiou, S.H.; et al. Cisplatin-selected resistance is associated with increased motility and stem-like properties via activation of STAT3/Snail axis in atypical teratoid/rhabdoid tumor cells. Oncotarget 2015, 6, 1750-1768. [CrossRef] [PubMed]

35. Van Schaeybroeck, S.; Kalimutho, M.; Dunne, P.D.; Carson, R.; Allen, W.; Jithesh, P.V.; Redmond, K.L.; Sasazuki, T.; Shirasawa, S.; Blayney, J.; et al. ADAM17-Dependent c-MET-STAT3 Signaling Mediates Resistance to MEK Inhibitors in KRAS Mutant Colorectal Cancer. Cell Rep. 2014, 7, 1940-1955. [CrossRef] [PubMed]

36. Ishii, Y.; Waxman, S.; Germain, D. Tamoxifen stimulates the growth of cyclin D1-Overexpressing breast cancer cells by promoting the activation of signal transducer and activator of transcription 3. Cancer Res. 2008, 68, 852-860. [CrossRef] 